\title{
Exclusive Orbital Involvement by Mycobacterium tuberculosis
}

\author{
Akshit Gakhar ${ }^{1}$, Anshul Arora ${ }^{2}$ \\ 1,2 Department of Radiology, MM. Institute of Medical Sciences \& Research, Mullana, Ambala, Haryana, India.
}

\section{INTRODUCTION}

Tuberculosis (TB) is an airborne infectious disease caused by Mycobacterium tuberculosis that generally acts on the lungs. However, extrapulmonary tubercular involvement of the eye and other orbital structures may occur. TB can affect nearly any tissue in the eye, and a high index of suspicion is required for accurate diagnosis. Early detection of the orbital TB is rare and can lead to loss of an affected eye. However, exclusive extrapulmonary involvement of the orbit without any pulmonary manifestations is very rare and I report one such case.

Tuberculosis is one of the causes of morbidity and mortality in the underdeveloped nations. ${ }^{1,2}$ Centers for Disease Control and Prevention (CDC) states that a large proportion of the world's population (1/3) is infected with tuberculosis. However, just one tenth of those infected will have symptoms of the disease. ${ }^{3}$ of these, $16-27 \%$ have extrapulmonary involvement, which includes those with orbital and external eye disease. ${ }^{4}$ Orbital Tuberculosis is rare, even in endemic areas. $^{5}$ Malignant lesions, cavernous malformations, venous varix, and nontuberculous infections and orbital pseudotumor are the lesions generally seen in youngsters. ${ }^{6}$ Orbital tuberculosis is generally seen in youngsters, affecting the girls more than the boys. ${ }^{7}$ The disease generally involves one of the orbit and advances slowly. It has a slow onset, with patient's clinical history dating back to months and years. Studies have shown that there is generally the involvement of left orbit.7

\section{PRESENTATION OF CASE}

A 37-year-old man came to the OPD (Out-Patient Department) with complaints of palpable swelling under left lower eyelid which was gradually increasing in size since 3 years. (Image A, B) He had no significant past history.

Patient got a CECT (Contrast-Enhanced Computed Tomography) head and face done for further evaluation of the lesion and it showed an enhancing soft tissue attenuation lesion along medial aspect of left orbit. (Image $C, D$ ) The mass was seen to cause destruction of medial wall of orbit and was seen abutting the eye ball and medial rectus and inferior oblique muscle laterally. No intraconal extension was seen. No involvement of the optic nerve was seen. Patient was advised contrast enhanced MRI (Magnetic Resonance Imaging) and histopathological correlation.

On USG (Ultra-Sono-Graphy) the lesion appeared as a soft tissue mass with no internal vascularity. (Image E, F)

On MRI the soft tissue mass appeared isointense on T1 (Image G) and mildly hypointense on T2W (Image $\mathrm{H}$ ) images seen in the inferomedial aspect of the left orbit near the medial canthus producing a focal bulge in the overlying skin and subcutaneous tissue. The lesion showed mild to moderate heterogenous enhancement in post contrast images. (Image I) Laterally the lesion was seen abutting the left globe with mild posterior extension into the extraconal compartment of left orbit. No intraconal extension was seen. Fat planes with the adjacent extra-ocular muscles were normal.
Corresponding Author: Dr. Akshit Gakhar, Department of Radiology, MM. Institute of Medical Sciences

\& Research, Mullana,

Ambala, Haryana, India.

E-mail: akshitgakhar07@gmail.com

DOI: $10.14260 /$ jemds/2020/835

How to Cite This Article:

Gakhar A, Arora A. Exclusive orbital involvement by mycobacterium tuberculosis. J Evolution Med Dent Sci 2020;9(50):3811-3814, DOI: 10.14260/jemds/2020/835

Submission 04-09-2020,

Peer Review 26-10-2020,

Acceptance 03-11-2020,

Published 14-12-2020.

Copyright (C) 2020 Akshit Gakhar et al. This is an open access article distributed under Creative Commons Attribution License [Attribution 4.0 International (CC BY 4.0)] 

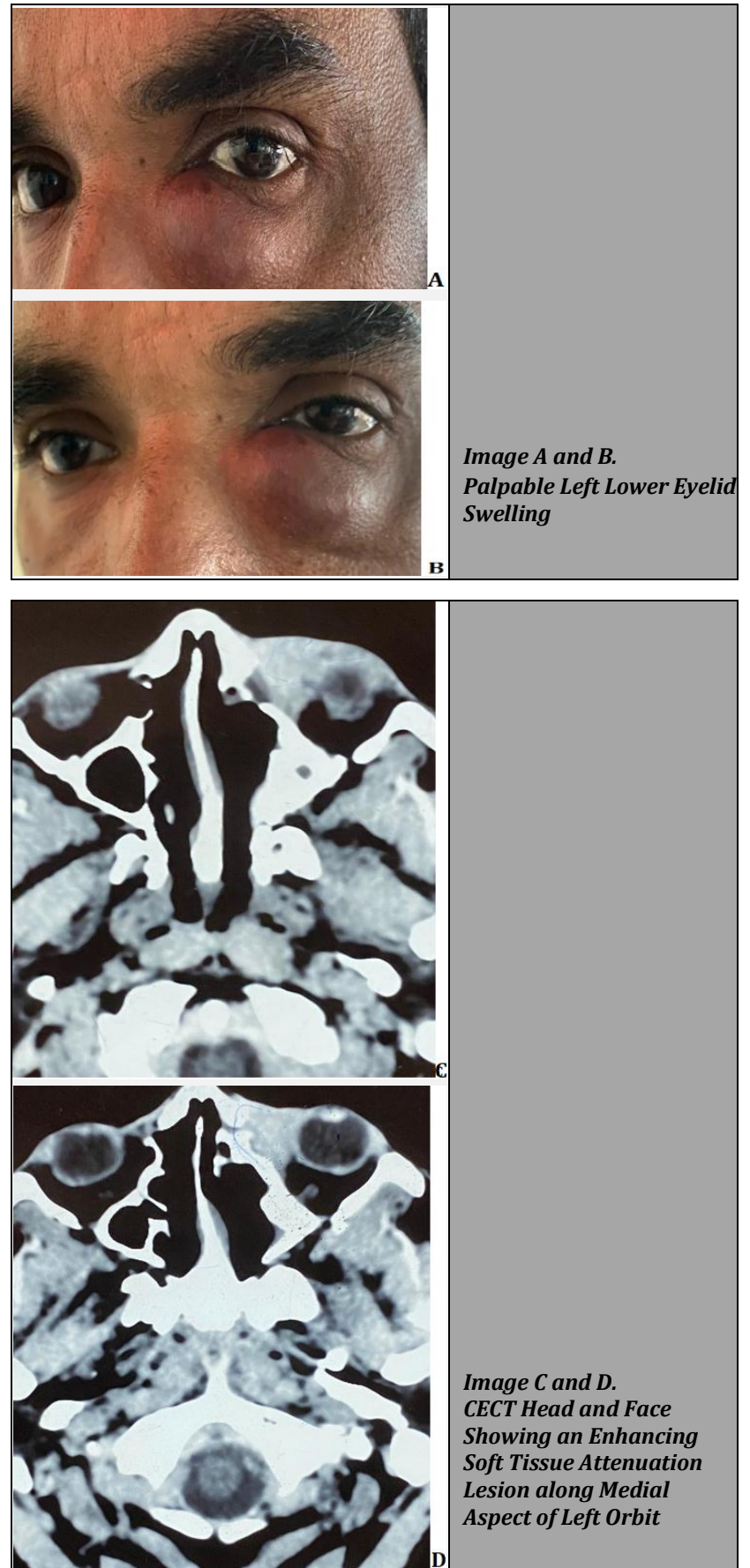

Image $C$ and $D$. CECT Head and Face Showing an Enhancing Soft Tissue Attenuation Lesion along Medial Aspect of Left Orbit
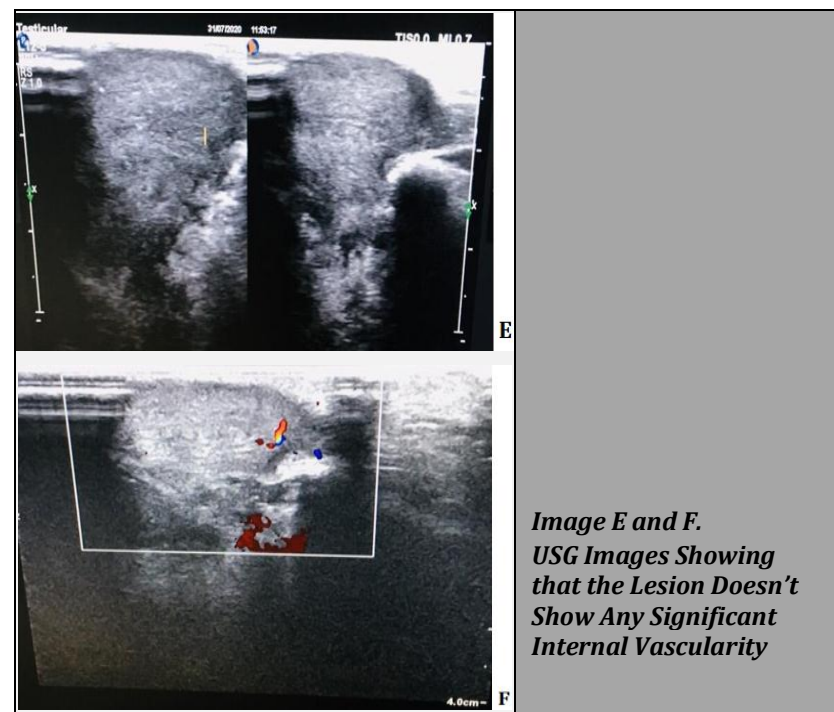

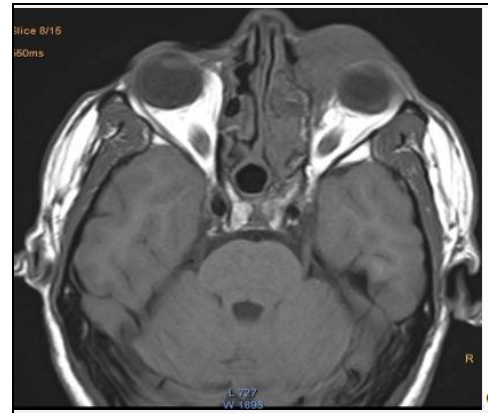

Image $\boldsymbol{G}$

Lesion Appearing

Isointense on T1W Image

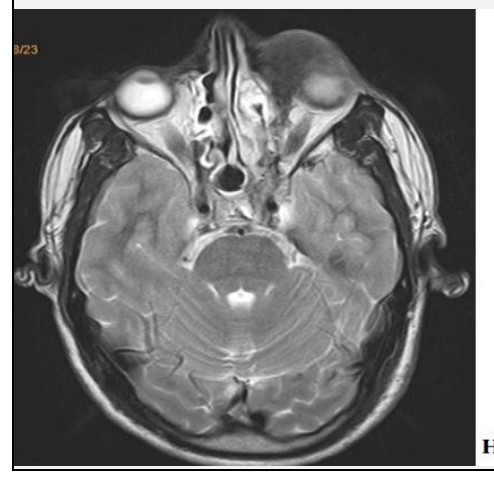

Image $\boldsymbol{H}$.

Lesion Appearing

Hypointense on T2W

Image

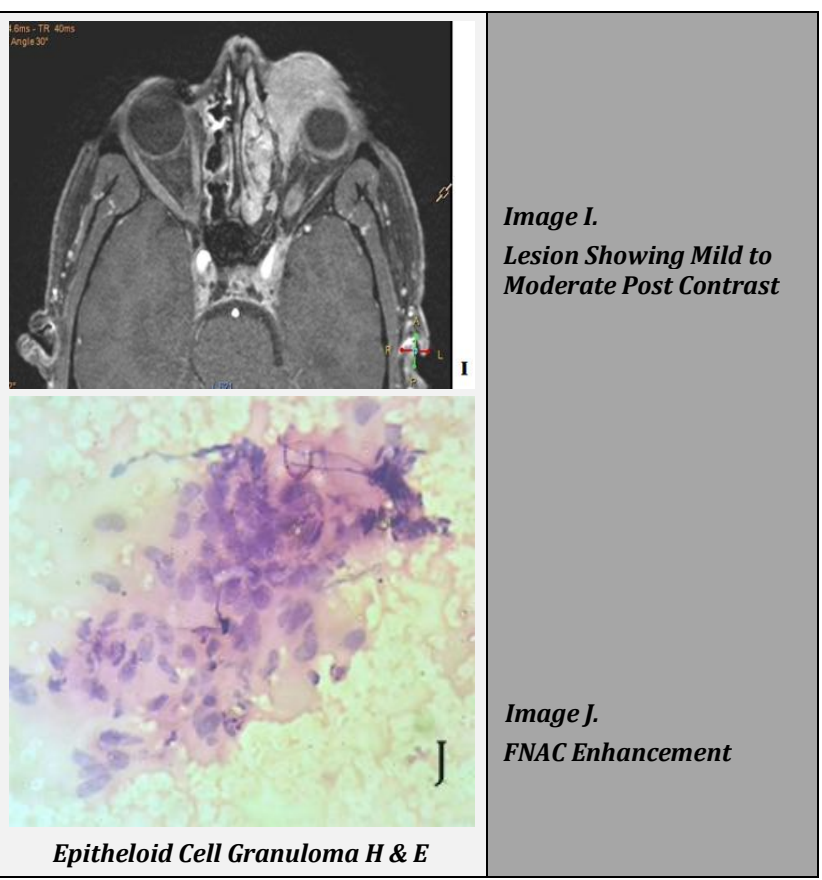

Medially the lesion was seen to cause the erosion of the lateral wall of ethmoid sinus / medial wall of orbit with minimal extension of soft tissue at medial aspect of left sided ethmoidal air cells. Inferiorly the lesion was showing tongue like extension into the nasolacrimal duct. No intracranial extension of the mass was seen. No particular diagnosis could be made on MRI as well and the patient was advised to get a FNAC (Fine Needle Aspiration Cytology) / biopsy done.

On FNAC the smears showed numerous epithelioid granulomas (Image J, K, L) along with multinucleated giant cells (Image $\mathrm{K}$ ) and few macrophages. It was confirmed that it is a tubercular granulomatous pathology.

On Chest X-ray AP view no evidence of pulmonary tuberculosis was found. (Image M) 

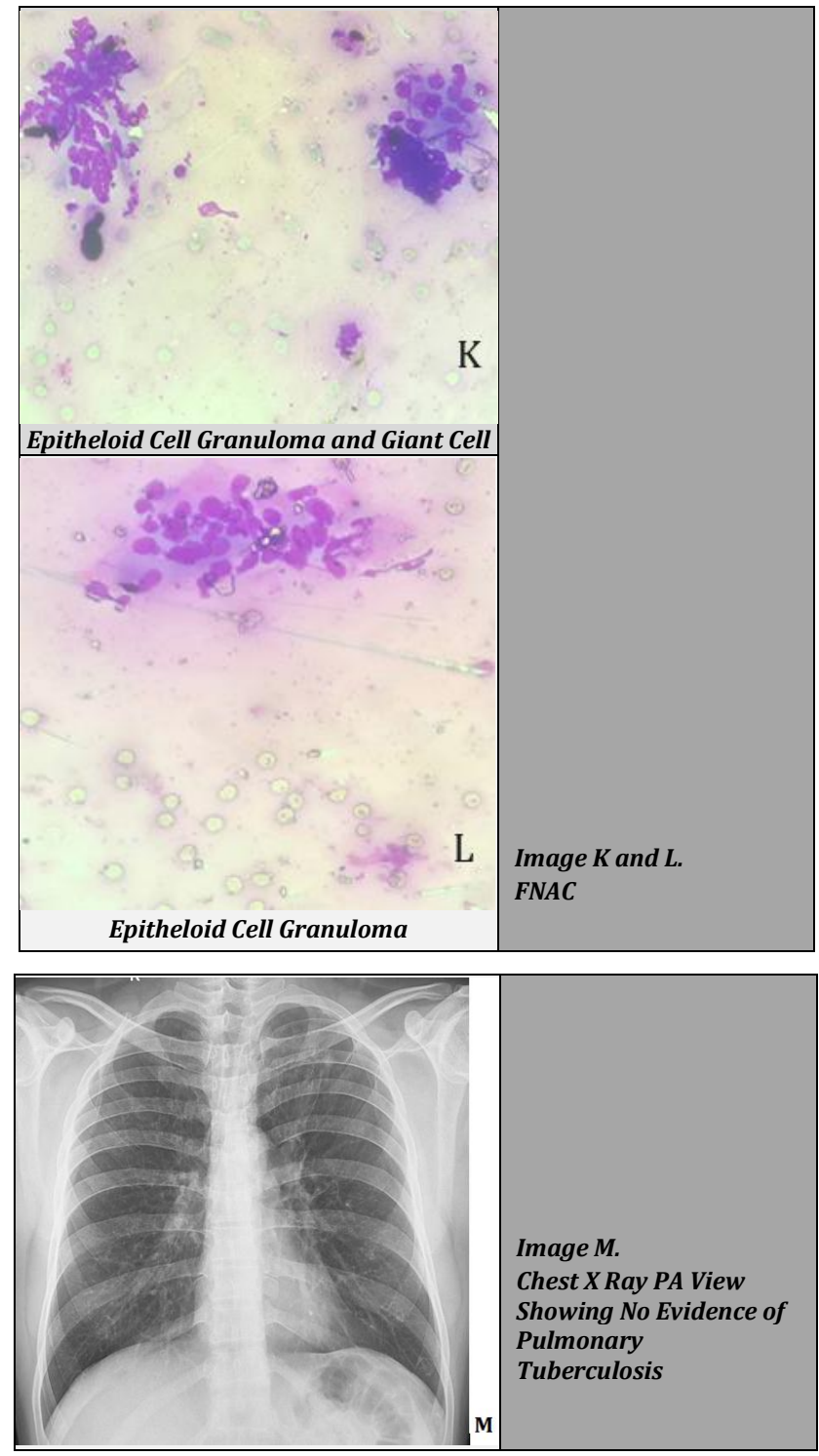

\section{DISCUSSION}

The primary tubercular manifestation is generally intrathoracic, but sites other than lungs, such as cervical lymph nodes or ileo-caecal intrabdominal disease, can be seen. ${ }^{7}$ Haematogenous spread via primary lesion or direct extension from affected opacified sinuses can involve the orbit. ${ }^{8}$ Orbital Tuberculosis generally occurs with bony erosions and destruction (usually the frontal and sphenoid bones), sclerosis may be seen. It can also lead to extraconal involvement with or without subperiosteal abscess formation. It can lead to intracranial extension via the infratemporal fossa. Lacrimal gland enlargement or abscess formation can also be one of the presenting feature. Lateral wall invasion or lesion generally suggests a haematogenous mode of spread however, the medial wall invasion generally suggests extension of infection from the adjacent opacified sinuses. ${ }^{8}$ Orbital Tuberculosis may occur singularly with orbital structure lesions. ${ }^{9}$

\section{Bony Involvement}

Orbital tuberculosis generally affects the bones of the orbital wall, i.e. the orbital plate of the frontal bone, the sphenoid, or the zygomatic bone. Extension into the sphenoid is also seen but is rare. ${ }^{7}$ Zygoma involvement along with lower lid involvement has been seen. ${ }^{10}$ Periosteal inflammation is the common occurrence of Tuberculosis involvement and generally affects the outer margin of the orbit.11 Bony involvement may also occur as mild irregularity in the cortex and invasion. Thickening and sclerotic margins of the bones represent chronicity of the disease. Other aetiologies of bony invasion in the young children are neuroblastoma, which generally does not present with associated subperiosteal abscess and Ewing sarcoma, which generally presents with onion ring periosteal reaction and a mass lesion.

\section{Extraconal Involvement}

Orbital as well as subperiosteal abscess generally present in extraconal location. Intraconal extension is generally very rare.

\section{Intracranial Extension}

Occurrence of orbital and CNS (Central Nervous System) Tuberculosis simultaneously has been seen, ${ }^{12}$ but ocular Tuberculosis with intracranial extension has also been seen which is seen in the form of abscess formation in the extradural location. $5,13,14$

\section{Lacrimal Gland Involvement}

Only lacrimal gland involvement has also been observed. ${ }^{15}$ It can occur in the form of its enlargement or abscess formation. Other aetiologies that may lead to lacrimal gland hypertrophy include lymphoma and sarcoidosis; however, these are generally seen with involvement on both sides and no abscess / bony invasion or destruction.

\section{Preseptal Thickening}

Due to extensive inflammation there may be thickening of the preseptal space.

\section{Treatment}

Tubercular cases are treated by both an ophthalmologist and a respiratory medicine specialist, as simultaneous management is needed to relieve the patients. Management with drugs for orbital tuberculosis is same as that for tuberculosis of the lung; although, recurrence of orbital tuberculosis is more frequent and may need a long course of management. ${ }^{16}$ The Center of disease control advices fourdrug treatment with isoniazid, rifampin, pyrazinamide and ethambutol for duration of 2 months, following which an extra 4- 7 months of treatment with isoniazid and rifampin. The period of treatment can be increased because of the usual slow response to treatment in orbital tuberculosis or in cases of multidrug resistance. ${ }^{13}$ In patients of multi-drug resistance recommended agents, rifabutin, fluoroquinolones, interferon gamma, and linezolid can also be used.

Financial or other competing interests: None.

Disclosure forms provided by the authors are available with the full text of this article at jemds.com. 


\section{REFERENCES}

[1] Helm CJ, Holland GN. Ocular tuberculosis. Surv Ophthalmol 1993;38(3):229-56.

[2] Schlernitzauer DA, Hodges FJ, Bagan M. Tuberculoma of the left optic nerve and chiasm. Arch Ophthalmol 1971;85(1):75-8.

[3] Gupta V, Gupta A, Rao NA. Intraocular tuberculosis -- an update. Surv Ophthalmol 2007;52(6):561-87.

[4] WHO. Global tuberculosis report 2013. Geneva: World Health Organization 2013.

[5] Oakhill A, Willshaw H, Mann JR. Unilateral proptosis. Arch Dis Child 1981;56(7):549-51.

[6] Sen DK. Tuberculosis of the orbit and lacrimal gland: a clinical study of 14 cases. J Pediatr Ophthalmol Strabismus 1980;17(4):232-8.

[7] Khalil M, Lindley S, Matouk E. Tuberculosis of the orbit. Ophthalmology 1985;92(11):1624-7.

[8] Raina UK, Jain S, Monga S, et al. Tubercular preseptal cellulitis in children: A presenting feature of underlying systemic tuberculosis. Ophthalmology 2004;111(2):2916.
[9] Pillai S, Malone TJ, Abad JC. Orbital tuberculosis. Ophthalmic Plast Reconstr Surg 1995;11(1):27-31.

[10] MacFaul PA. The ocular adnexa: lacrimal, orbital and paraorbital diseases. Part 2. In: Duke ES. System of ophthalmology. Vol. 13. London: Henry Kimpton 1974:902-5.

[11] De Castro CC, De Barros NG, Campos ZM, et al. CT scans of cranial tuberculosis. Radiol Clin North Am 1995;33(4):753-69.

[12] Gupta V, Angra P. Orbital tubercular abscess with intracranial extension. Curr Ophthalmol 1995:201-3.

[13] Dewan T, Sangal K, Premsagar IC, et al. Orbital tuberculoma extending into the cranium. Ophthalmologica 2006;220(2):137-9.

[14] Mortada A. Tuberculoma of the orbit and lacrimal gland. Br J Ophthalmol 1971;55(8):565-7.

[15] Patel SS, Saraiya NV, Tessler HH, et al. Mycobacterial ocular inflammation: delay in diagnosis and other factors impacting morbidity. JAMA Ophthalmol 2013;131(6):752-8.

[16] American Thoracic Society, CDC, Infectious Diseases Society of America. Treatment of tuberculosis. MMWR Recomm Rep 2003;51(RR-11):1-77. 